\title{
SPATIOTEMPORAL GAIT AND CENTRE OF MASS VARIABLES WHILE PERFORMING DIFFERENT SMARTPHONE TASKS AND CONFRONTING OBSTACLE AMONG YOUNG ADULTS
}

original paper

() Wroclaw University of Health and Sport Sciences

DOI: https://doi.org/10.5114/hm.2022.107975

\section{TAUFIK EKO SUSILO ${ }^{\circledR}$, SUNEE BOVONSUNTHONCHAI ${ }^{\circledR}$, PEEMONGKON WATTANANON $^{\circledR}$}

Faculty of Physical Therapy, Mahidol University, Nakhon Pathom, Thailand

\section{ABSTRACT}

Purpose. Smartphone is one of the essential tools but may be inappropriate during locomotion or transportation owing to cognitive distractions. The study aimed to investigate the main effects of smartphone tasks, obstacle conditions, and their interaction on the spatiotemporal gait and centre of mass $(\mathrm{COM})$ variables among healthy young adults.

Methods. The study used a single group with repeated measures design. Overall, 20 participants completed 4 smartphone tasks (no task, texting, calling, and watching), combined with 2 conditions of obstacle confrontation (with and without). Spatiotemporal gait (step length, step time, cadence, and gait speed) and COM variables during gait (excursion and velocity in mediolateral and vertical directions) were collected.

Results. Significant effects of smartphone tasks and obstacle conditions were found, while no interaction effect between smartphone tasks and obstacle was found. There were alterations of the spatiotemporal gait and COM variables during walking, both with and without obstacle. The obstacle condition significantly influenced the different tasks of mobile perturbation, all spatiotemporal gait and COM variables, except for the COM mediolateral velocity.

Conclusions. Confronting smartphone tasks and obstacle conditions concurrently challenges young adults to adjust their movement and balance control systems to perform the tasks successfully.

Key words: smartphone, obstacle crossing, walking, spatiotemporal, centre of mass

\section{Introduction}

Presently, the smartphone has become a practical item for every need of human activities. It has been developed to serve a broad spectrum of functions and abilities. However, smartphone use may be prone to addiction, especially among children and emerging adults [1]. In particular, the young adult generation comprises the majority of users who usually perceive smartphone as the most crucial part of their lifestyles for socializing, work, and entertainment [2-4]. Combining a gyroscope and accelerator inside the phone makes it possible to measure human motion efficiently [5]. However, most tasks performed on the smartphone in daily life include texting, calling, and watching, and they are often accompanied by other locomotor activities [6, 7]. This dual-task activity leads to the risk of injury from a collision, fall, or crash $[2,6,7]$.
For walking, previous studies have revealed disturbance when using smartphones concerning the primary gait performance in public areas. It comprises slow walking speed, increased variability, lack of awareness, and late initiation for crossing roads [8,9]. This reduced performance results from cognitive loading that distracts the sensory distribution from prioritizing the tasks because of performing double activities in parallel [10]. Then, it engages the executive function concerning the cognitive process while obtaining information when walking [11]. By perturbing the sensory input, the central pattern generator alternates the rhythmic flexor and extensor activity regarding spinal locomotion [12]; as a result, biomechanical changes occur when walking while performing dualtask conditions. Regarding its interference with memory load and causing errors, examining how different smartphone tasks influence gait and balance would be important.

Correspondence address: Sunee Bovonsunthonchai, Faculty of Physical Therapy, Mahidol University, 999 Phuttamonthon Sai 4 Rd., Salaya, Phuttamonthon, Nakhon Pathom, 73170, Thailand, e-mail: sunee.bov@mahidol.edu, https://orcid.org/0000-0003-1044-3174

Received: April 24, 2020

Accepted for publication: February 8, 2021

Citation: Susilo TE, Bovonsunthonchai S, Wattananon P. Spatiotemporal gait and centre of mass variables while performing different smartphone tasks and confronting obstacle among young adults. Hum Mov. 2022;23(3):92-103; doi: https://doi. $\operatorname{org} / 10.5114 / \mathrm{hm} .2022 .107975$. 
The cyclical motion of the limbs while walking results in the centre of mass (COM) displacement in the direction of body motion. A key to the clinical gait analysis concept has been proposed to be associated with energy conservation and COM movement [13]. The COM motion in the mediolateral and vertical directions helps evaluate balance control during gait and is often used as an indicator of strategy adaptation to different tasks or environmental contexts [12]. The risk of injury from loss of balance is significantly increased, especially with challenging tasks, such as walking on an uneven footpath or slippery surface, and walking and crossing the door sill or other obstacles.

Most related studies considered only the texting task on the smartphone [9, 14-16]. These findings may not provide a comprehensive understanding of the various contexts of smartphone use regarding consumer behaviour in modern times. Therefore, the study aimed to examine the main effects of different smartphone tasks (no task, texting, calling, and watching) and obstacle conditions (with and without), as well as their interaction, on gait performance and COM among healthy young adults. We hypothesized that smartphone tasks and obstacle conditions could significantly affect spatiotemporal gait and COM variables.

\section{Material and methods}

\section{Design and participants}

The study involved a single group with repeated measures design. The participants were informed about the research details and protocols. They were recruited from healthy young adults from Mahidol University by posters at the student information board and digital flyers in the social media. The inclusion criteria were as follows: (1) age of 18-35 years, (2) right hand and leg dominance tested with the Edinburgh Handedness Inventory-Short Form and the Waterloo Footedness Questionnaire-Revised, (3) experience in using a touchscreen smartphone for at least 1 year, (4) clear vision, and (5) ability to understand English both written and spoken. Participants were excluded if they had: (1) musculoskeletal impairments such as sprain or fracture, (2) history of neurological deficits, (3) leg discrepancy $\geq 2 \mathrm{~cm}$, (4) alcohol consumption within 24 hours, (5) obesity with body mass index of $\geq 25 \mathrm{~kg} / \mathrm{m}^{2}$, (6) dizziness or vertigo, or (7) any medical treatment. Participants who passed the selection criteria were investigated for the demographic data and smartphone using details before data collection.

\section{Data collection protocol}

Gait performance while using a smartphone was recorded repeatedly in 2 obstacle conditions (with and without) by using 10 high-speed cameras (Vantage series, Vicon ${ }^{\mathrm{TM}}$ motion analysis system; Oxford Metrics Ltd., Oxford, UK) synchronized with 2 force plates (series: OR6 7-4000; Advanced Mechanical Technology, Inc., Watertown, MA, USA). The data were captured at $100 \mathrm{~Hz}$ for kinematic and $1000 \mathrm{~Hz}$ for kinetic analysis. A set of 39 reflective markers were attached to bony landmarks in accordance with a fullbody plug-in-gait model. Two additional markers were placed on the border of an obstacle (size: $40 \times 15 \times$ $6 \mathrm{~cm}$ in width, length, and depth).

The participants walked over an 8-meter walkway while using a smartphone (Samsung Galaxy J7, version 2 , Korea) prepared by a researcher to perform different smartphone tasks (texting, calling, and watching), in 2 obstacle conditions (with and without). The sequence of testing the smartphone tasks was randomized with the Random UX number generator (version 2.1.9, Google Play). In each smartphone task, the subjects were recorded with no obstacle first, and then when crossing over an obstacle placed in the middle of the walkway. Three successive trials of walking were captured for each of the smartphone tasks and obstacle conditions. Three different questions and details were used for each trial in order to avoid familiarization with the testing protocol. The details for texting, calling, and watching are described in Table 1.

\section{Data processing}

Gait data were processed by using the Nexus software, version 2.7.1. The selected time frame of gait was in the middle of the walkway, and gait events were identified by foot placement over one gait cycle for each leg, left and right. Data were filtered with the $4^{\text {th }}$ order low-pass Butterworth technique with a cutoff frequency for marker trajectory at $4 \mathrm{~Hz}$ and analogue at $25 \mathrm{~Hz}$. Gait performance was digitized for the spatiotemporal gait and COM variables and exported as ASCII files. The averaged data of 3 trials of walking were used for further analysis.

\section{Outcome variables}

The spatiotemporal gait variables comprised step length (m), step time (s), cadence (steps/min), and gait speed $(\mathrm{m} / \mathrm{s})$. The step variables were computed for dis- 


\section{HUMAN MOVEMENT}

T.E. Susilo, S. Bovonsunthonchai, P. Wattananon, Smartphone use and variables of gait and centre of mass

Table 1. Details of smartphone tasks

\begin{tabular}{|c|c|c|c|}
\hline \multirow{2}{*}{$\begin{array}{l}\text { Smartphone } \\
\text { task }\end{array}$} & \multirow{2}{*}{ Instruction details } & \multicolumn{2}{|c|}{ Questions used for checking } \\
\hline & & Without obstacle & With obstacle \\
\hline Texting & $\begin{array}{l}\text { Text simple sentences, } \\
\text { including a standard sentence } \\
\text { with a similar amount of words } \\
\text { and symbols }\end{array}$ & $\begin{array}{l}2^{\text {nd }} \text { October } 1990 \\
\text { Do you live in Bangkok? } \\
\text { The location is at faculty, } 6^{\text {th }} \text { floor }\end{array}$ & $\begin{array}{l}5^{\text {th }} \text { November } 1991 \\
\text { Do you live in Salaya? } \\
\text { The location is at library, } 3^{\text {rd }} \text { floor }\end{array}$ \\
\hline Calling & $\begin{array}{l}\text { Answer a simple question } \\
\text { by smartphone }\end{array}$ & $\begin{array}{l}\text { Where are you from? } \\
\text { Please calculate } 100-7 \\
\text { What is the colour of the flag } \\
\text { of Thailand? }\end{array}$ & $\begin{array}{l}\text { Where is the location of Mahidol } \\
\text { Uni? } \\
\text { Please calculate } 86-7 \\
\text { What is the colour of the flag } \\
\text { of your country? }\end{array}$ \\
\hline Watching & $\begin{array}{l}\text { Watch a video clip while } \\
\text { walking and answer the } \\
\text { questions about the video } \\
\text { at the end of each walking trial }\end{array}$ & $\begin{array}{l}\text { What is the food that appears } \\
\text { during a typhoon? } \\
\text { What is the colour of long larva? } \\
\text { How many animals were trapped } \\
\text { in the spider's network? }\end{array}$ & $\begin{array}{l}\text { What is the main scene in the video? } \\
\text { What is the colour of short larva? } \\
\text { What is the season during the video? }\end{array}$ \\
\hline
\end{tabular}

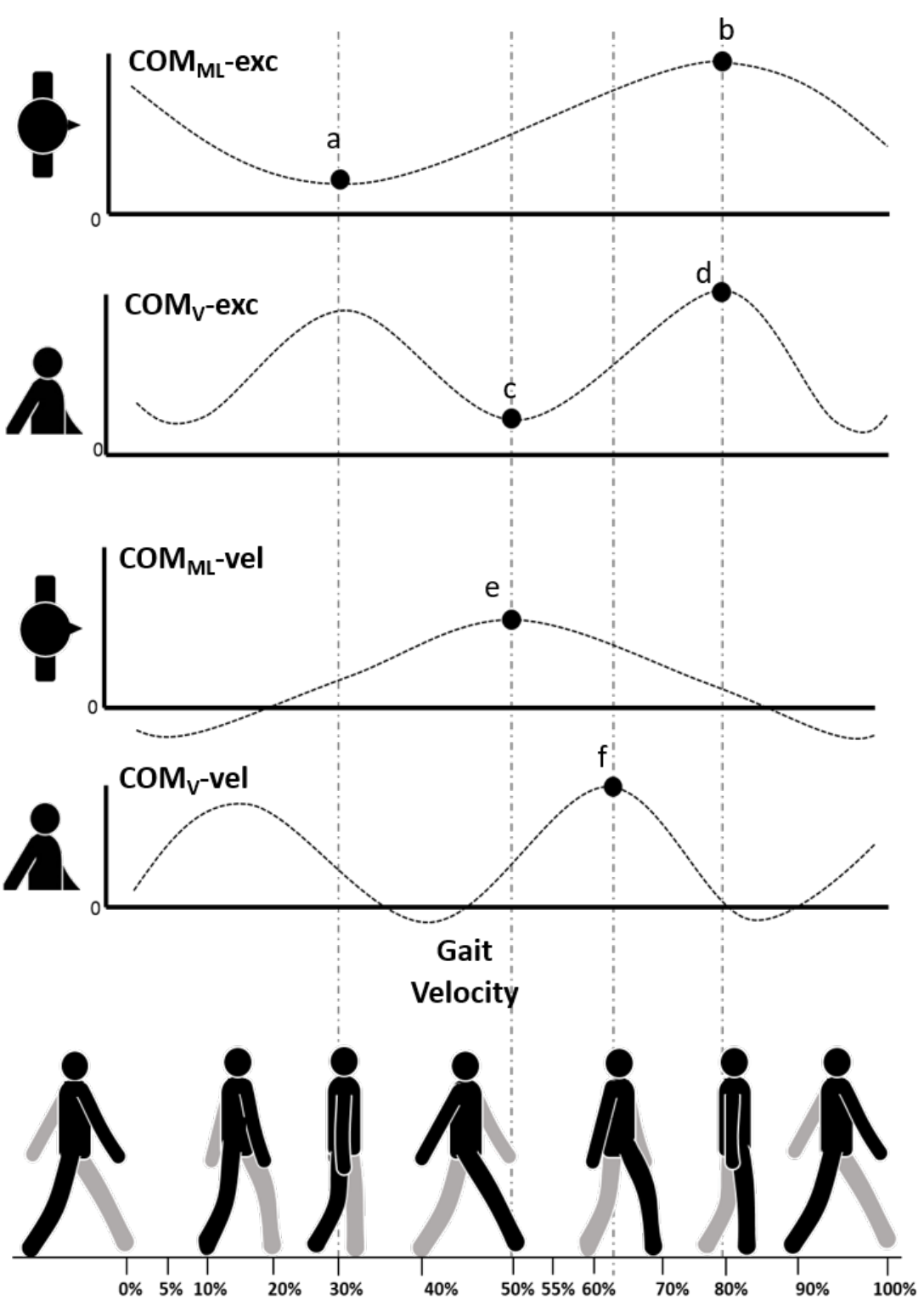
COM - centre of mass
$\mathrm{ML}$ - mediolateral direction
$\mathrm{V}-$ vertical direction
exc - excursion
vel - velocity
$\mathrm{a}-$ minimum excursion of COM in the mediolateral direction
$\mathrm{b}$ - maximum excursion of COM in the mediolateral direction
$c$ - minimum excursion of $\mathrm{COM}$ in the vertical direction
$d$ - maximum excursion of COM in the vertical direction
e - peak velocity of COM in the mediolateral direction
$f$ - peak velocity of COM in the vertical direction


tance and time from heel to opposite heel markers. Cadence comprised the number of steps taken within one minute, while gait speed was computed from the total stride length divided by stride time.

The COM variables were extracted for the COM excursion (COM-exc) and COM velocity (COM-vel) in the mediolateral (ML) and vertical (V) directions. All $\mathrm{COM}$ variables were plotted over the gait cycle and represented sinusoidal patterns, as shown in Figure 1 . The 2 peak points (maximum and minimum) were identified and calculated for COM-exc, and a single peak point (maximum) was identified for COM-vel.

For $\mathrm{COM}_{\mathrm{ML}}$-exc, the 2 peak points appeared during the single-limb support (30\% gait cycle) and midswing ( $30 \%$ gait cycle). For $\mathrm{COM}_{V}$-exc, the 2 peak points appeared during the double-limb support (50\% gait cycle) and midswing ( $80 \%$ gait cycle). The peak velocities of $\mathrm{COM}_{\mathrm{ML}}$-vel and $\mathrm{COM}_{\mathrm{V}}$-vel were selected during the double-limb support (50\% gait cycle) and early swing (65\% gait cycle), respectively.

\section{Data analysis}

Data were analysed by using the SPSS software, version 23.0, with a significance level set at $p<0.05$. The Shapiro-Wilk test showed normal distribution. A $4 \times 2$ (smartphone tasks and obstacle conditions) repeated measures ANOVA was used to determine the effect of smartphone tasks (no task, texting, calling, and watching), the effect of obstacle conditions (with and without an obstacle), and the interaction effect of smartphone tasks and obstacle conditions concerning the spatiotemporal gait and COM variables. For further analysis, the paired $t$-test was applied to compare the data between obstacle conditions in each smartphone task, and one-way repeated measures ANOVA served to compare the data between 4 different smartphone tasks in each obstacle condition.

\section{Sample size calculation}

The sample size was calculated in our pilot study $(n=10)$ on gait speed. The 4 smartphone tasks (no task, texting, calling, and watching) and 2 obstacle conditions (with and without obstacle) were defined as within- and between-subject factors. The partial eta squared $\left(\eta^{2}\right)$ for within-subject factor, between-subject factor, and the correlation between tasks equalled 0.267, $0.213,0.562$, respectively. The sample size was estimated by using the $G^{*}$ Power software, version 3.1.9.2, with the function for the F-test repeated measures ANOVA between- and within-subject, alpha proba- bility of 0.05 , power of 0.80 , and separate computed functions for between- and within-subject factors testing. The estimated sample size for between- and within-subject factors was 18 and 8. Therefore, a total of 20 participants was sufficient.

\section{Ethical approval}

The research related to human use has complied with all the relevant national regulations and institutional policies, has followed the tenets of the Declaration of Helsinki, and has been approved by the Mahidol University Central Institutional Research Board (MUCIRB 2019/233.0609) and registered in the Thai Clinical Trials Registry (TCTR20191110002).

\section{Informed consent}

Informed consent has been obtained from all individuals included in this study.

\section{Results}

The characteristics of the participants and smartphone use details are demonstrated in Table 2. Twenty healthy young adults comprising 11 females and 9 males participated in the study. The subjects' mean age was $24.45 \pm 3.80$ years, the duration of smartphone possession equalled $2.50 \pm 1.24$ years, and smartphone was used for $12.25 \pm 3.81$ hours per day.

Table 2. Demographic data of the participants and smartphone use details

\begin{tabular}{lc}
\hline Variable & $\begin{array}{c}\text { Value } \\
(n \text { or mean } \pm S D)\end{array}$ \\
\hline Sex: female/male $(n)$ & $11 / 9$ \\
Age (years) & $24.45 \pm 3.80$ \\
Body mass $(\mathrm{kg})$ & $57.53 \pm 5.96$ \\
Body height $(\mathrm{cm})$ & $166.75 \pm 7.48$ \\
Body mass index $\left(\mathrm{kg} / \mathrm{m}^{2}\right)$ & $20.70 \pm 2.02$ \\
Smartphone possession duration (years) & $2.50 \pm 1.24$ \\
Smartphone use (hours/day) & $12.25 \pm 3.81$ \\
\hline
\end{tabular}

Effect of smartphone tasks and obstacle conditions and the interaction effect between smartphone task and obstacle condition on the spatiotemporal gait and COM variables

Significant main effects were found regarding smartphone task and obstacle condition $(p<0.05)$, but no interaction effect was observed between them $(p>0.05)$ in any spatiotemporal gait variable. For step length, the main effect of smartphone task $\left(\mathrm{F}_{(3,152)}=2.982\right.$, 
T.E. Susilo, S. Bovonsunthonchai, P. Wattananon, Smartphone use and variables of gait and centre of mass

$p=0.033)$ and main effect of obstacle condition $\left(\mathrm{F}_{(1,152)}=\right.$ 4.985, $p=0.027$ ) were determined, but no interaction effect was observed between smartphone task and obstacle condition $\left(\mathrm{F}_{(3,152)}=0.249, p=0.862\right)$. Concerning step time, the main effect of smartphone task $\left(\mathrm{F}_{(3,152)}=4.553, p=0.004\right)$ and main effect of obstacle condition $\left(\mathrm{F}_{(1,152)}=193.452, p<0.001\right)$ were found, but no interaction effect was observed between smartphone task and obstacle condition $\left(\mathrm{F}_{(3,152)}=0.565\right.$, $p=0.639)$. For cadence, the main effect of smartphone task $\left(\mathrm{F}_{(3,152)}=5.256, p=0.002\right)$ and obstacle condition $\left(\mathrm{F}_{(1,152)}=88.374, p<0.001\right)$ were noted, but no interaction effect was observed between smartphone task and obstacle condition $\left(\mathrm{F}_{(3,152)}=0.118, p=0.950\right)$. Regarding gait speed, the main effect of smartphone task $\left(\mathrm{F}_{(3,152)}=5.793, p=0.001\right)$ and main effect of obstacle condition $\left(\mathrm{F}_{(1,152)}=68.086, p<0.001\right)$ were established, but no interaction effect was observed between smartphone task and obstacle condition $\left(\mathrm{F}_{(3,152)}=0.075\right.$, $p=0.974)$.

Significant main effects of smartphone task and obstacle condition were found in all COM variables $(p<0.05)$, except for $\mathrm{COM}_{\mathrm{V}}$-vel, showing no effect of smartphone task $(p>0.05)$. In addition, no interaction effect was observed between smartphone task and obstacle condition in any COM variable $(p>0.05)$. For $\mathrm{COM}_{\mathrm{ML}}$-exc, the main effect of smartphone task $\left(\mathrm{F}_{(3,152)}\right.$ $=7.279, p<0.001)$ and main effect of obstacle condition $\left(\mathrm{F}_{(1,152)}=25.810, p<0.001\right)$ were determined, but no interaction effect was observed between smartphone task and obstacle condition $\left(\mathrm{F}_{(3,152)}=0.441, p=\right.$ 0.724). $\mathrm{COM}_{\mathrm{V}}$-exc presented the main effect of smartphone task $\left(\mathrm{F}_{(3,152)}=2.715, p=0.047\right)$ and main effect of obstacle condition $\left(\mathrm{F}_{(1,152)}=177.023, p<0.001\right)$, but no interaction effect between them $\left(\mathrm{F}_{(3,152)}=1.430\right.$, $p=0.236)$ was found. For $\mathrm{COM}_{\mathrm{ML}}$-vel, the main effects of smartphone task $\left(\mathrm{F}_{(3,152)}=5.073, p=0.002\right)$ and obstacle condition $\left(\mathrm{F}_{(1,152)}=9.563, p=0.002\right)$ were found, but no interaction effect was observed between them $\left(\mathrm{F}_{(3,152)}=0.892, p=0.447\right)$. For $\mathrm{COM}_{\mathrm{V}}$-vel, the main effects of smartphone task $\left(\mathrm{F}_{(3,152)}=0.130, p=0.942\right)$ and obstacle condition $\left(\mathrm{F}_{(1,152)}=35.654, p<0.001\right)$ were noted, but no interaction effect was established between them $\left(\mathrm{F}_{(3,152)}=0.395, p=0.756\right)$.

Comparisons of the spatiotemporal gait and COM variables between 4 smartphone tasks in each of the obstacle conditions

Table 3 and Figure 2 show the comparison of the spatiotemporal gait and COM variables between 4 smartphone tasks. During no obstacle condition, sig- nificant differences were found in step length $\left(\mathrm{F}_{(3,57)}=\right.$ $10.198, p<0.001)$, step time $\left(\mathrm{F}_{(3,57)}=10.643, p<0.001\right)$, cadence $\left(\mathrm{F}_{(3,57)}=11.049, p<0.001\right)$, gait speed $\left(\mathrm{F}_{(3,57)}=\right.$ 13.374, $p<0.001), \mathrm{COM}_{\mathrm{ML}}-\mathrm{exc}\left(\mathrm{F}_{(3,57)}=5.892, p=\right.$ $0.004)$, and $\mathrm{COM}_{\mathrm{ML}}-\operatorname{vel}\left(\mathrm{F}_{(2.111,40.116)}=5.561, p=0.007\right)$ between 4 smartphone tasks. In turn, no significant differences of $\mathrm{COM}_{\mathrm{V}}$-exc $\left(\mathrm{F}_{(2.020,38.380)}=2.158, p=0.129\right)$ or $\mathrm{COM}_{\mathrm{V}}$-vel $\left(\mathrm{F}_{(1.808,34.353)}=2.148, p=0.136\right)$ were observed.

Pairwise comparisons of the spatiotemporal gait and COM variables between 4 smartphone tasks are presented in Table 4 . For step length, significant differences were found between no task and texting ( $p=$ $0.001)$ and no task and watching $(p=0.010)$. Concerning step time, significant differences were observed between no task and texting $(p=0.001)$, no task and watching ( $p=0.006)$, and texting and calling ( $p=$ 0.016). For cadence, significant differences were determined between no task and texting $(p=0.001)$ and no task and watching ( $p=0.003)$. Regarding gait speed, significant differences were established between no task and texting $(p<0.001)$, no task and watching $(p=0.011)$, and texting and calling $(p=0.006)$. For $\mathrm{COM}_{\mathrm{ML}}-\mathrm{exc}$, there were significant differences between no task and calling $(p=0.048)$ and no task and texting ( $p=0.001)$. Concerning $\mathrm{COM}_{\mathrm{ML}}$-vel, significant differences were noted between no task and calling ( $p=0.008)$ and no task and texting ( $p=0.001)$.

In walking under obstacle condition (Table 3 and Figure 2), significant differences were found in step length $\left(\mathrm{F}_{(3,57)}=3.609, p=0.019\right)$, step time $\left(\mathrm{F}_{(3,57)}=\right.$ $6.744, p=0.001)$, cadence $\left(\mathrm{F}_{(2.214,42.068)}=6.688, p=\right.$ $0.002)$, gait speed $\left(\mathrm{F}_{(3,57)}=6.405, p=0.001\right), \mathrm{COM}_{\mathrm{ML}}-\mathrm{exc}$ $\left(\mathrm{F}_{(3,57)}=6.093, p=0.001\right), \mathrm{COM}_{\mathrm{V}^{-}} \operatorname{exc}\left(\mathrm{F}_{(3,57)}=9.288\right.$, $p<0.001)$, and $\mathrm{COM}_{\mathrm{ML}}-\operatorname{vel}\left(\mathrm{F}_{(3,57)}=4.099, p=0.011\right)$ between 4 smartphone tasks. Meanwhile, no main effect of $\mathrm{COM}_{\mathrm{V}}$-vel $\left(\mathrm{F}_{(3,57)}=0.458, p=0.713\right)$ was observed.

The pairwise comparisons showed significant differences in the variables between smartphone tasks while walking with an obstacle (Table 4). For step time, significant differences were determined between no task and calling ( $p=0.028)$, no task and texting ( $p=$ $0.009)$, and no task and watching ( $p=0.023$ ). For cadence, significant differences were established between no task and calling ( $p=0.003)$, no task and texting $(p=0.004)$, and no task and watching ( $p=0.008)$. Concerning gait speed, significant differences were found between no task and texting $(p=0.002)$ and no task and watching $(p=0.030)$. For $\mathrm{COM}_{\mathrm{ML}}-\mathrm{exc}$, significant differences were noted between no task and texting $(p=0.048)$ and no task and watching $(p=0.001)$. For $\mathrm{COM}_{\mathrm{V}}$-exc, there were significant differences between 


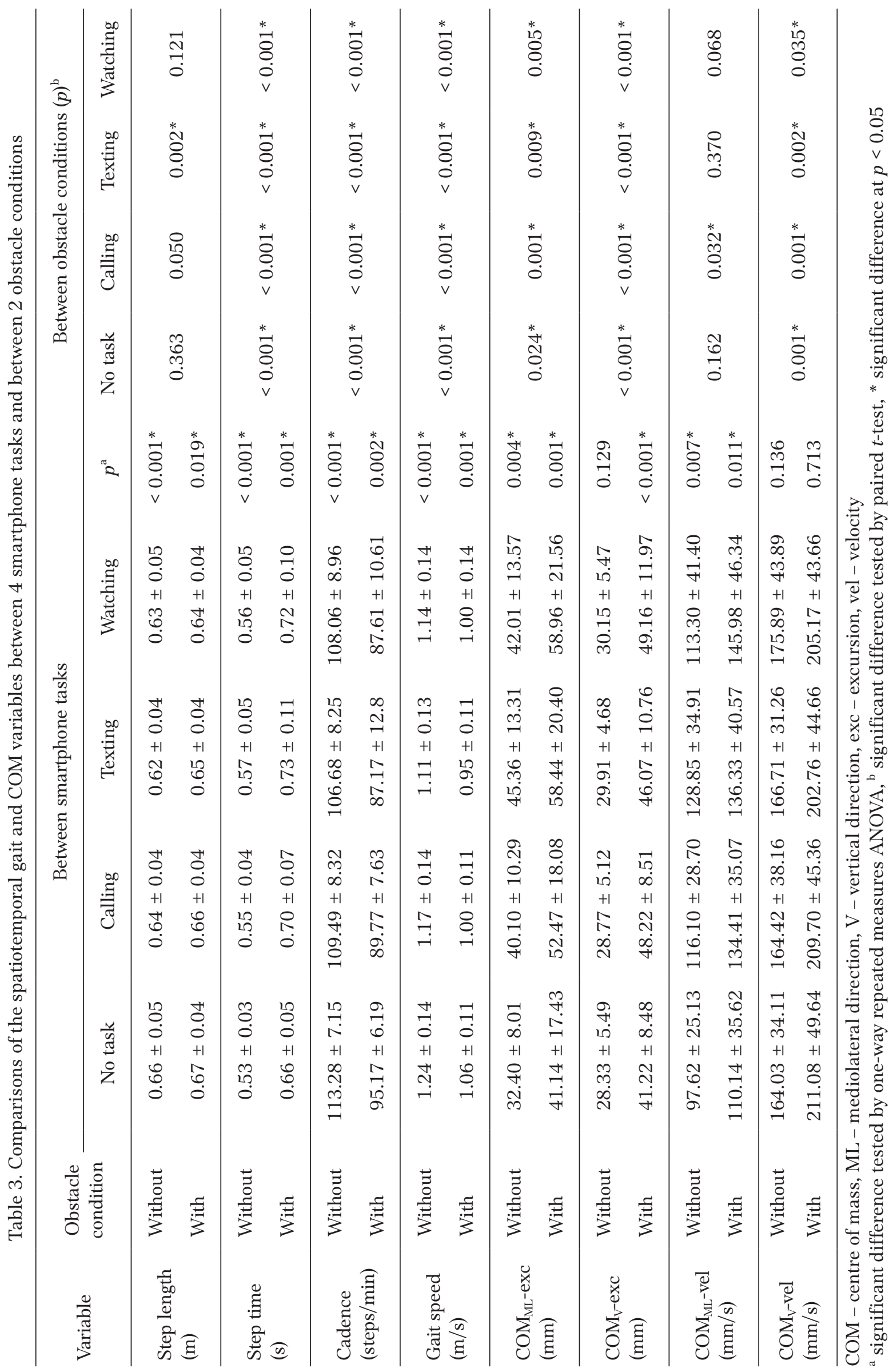




\section{HUMAN MOVEMENT}

T.E. Susilo, S. Bovonsunthonchai, P. Wattananon, Smartphone use and variables of gait and centre of mass
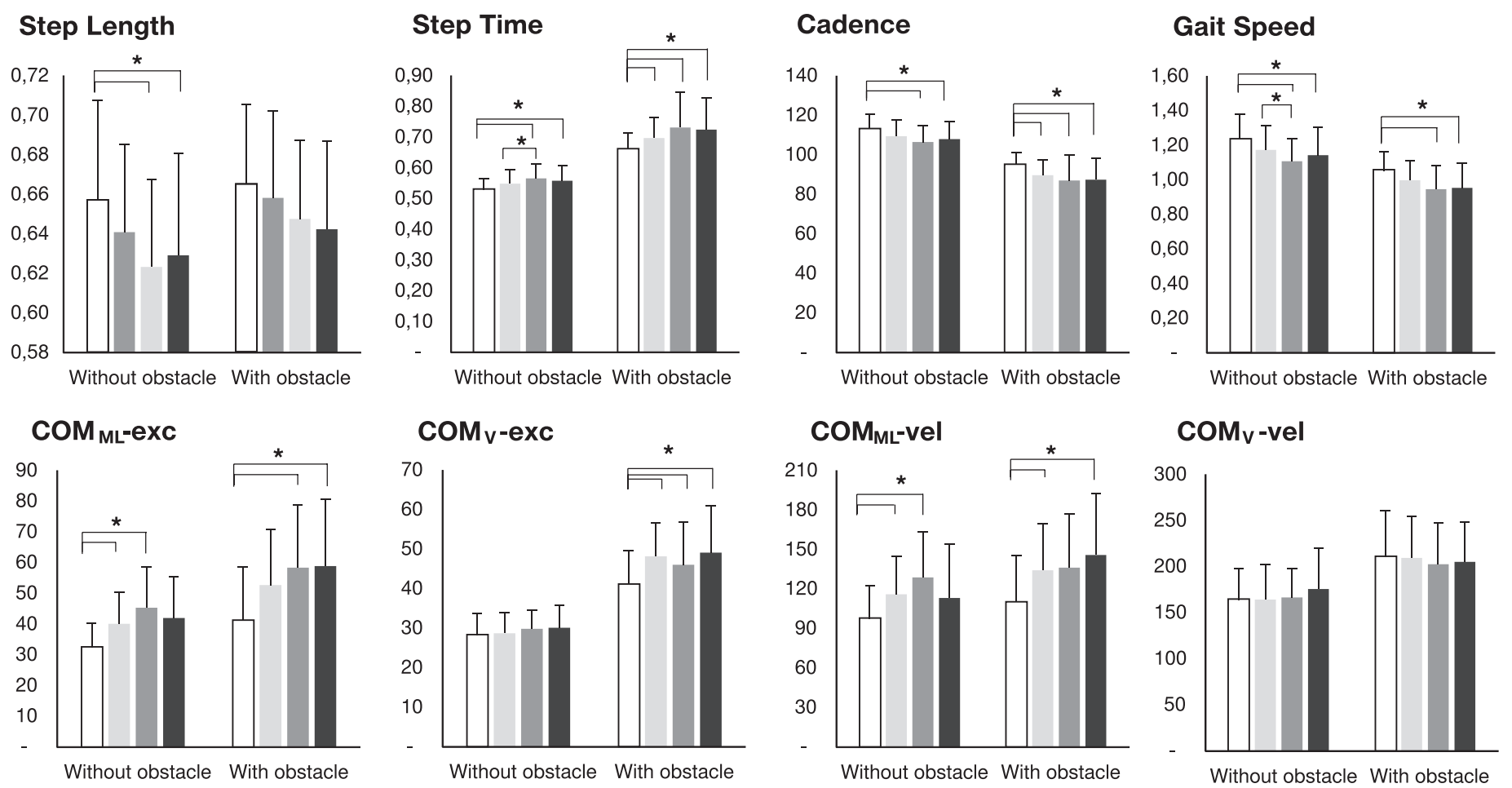

$\square$ No task Calling Texting

Watching

$\mathrm{COM}$ - centre of mass, $\mathrm{ML}$ - mediolateral direction, $\mathrm{V}$ - vertical direction, exc - excursion, vel - velocity

Unit of step length is $\mathrm{mm}$, step time is $\mathrm{s}$, cadence is steps $/ \mathrm{min}$, gait speed is $\mathrm{m} / \mathrm{s}, \mathrm{COM}_{\mathrm{ML}}$-exc and $\mathrm{COM}_{\mathrm{V}}$-exc is $\mathrm{mm}, \mathrm{COM}_{\mathrm{ML}}-\mathrm{vel}$ and $\mathrm{COM}_{\mathrm{v}}$-vel is $\mathrm{mm} / \mathrm{s}$.

${ }^{*} p<0.05$, pairwise comparison tested by the post-hoc Bonferroni

Figure 2. Bar charts of data between 4 smartphone tasks

Table 4. Pairwise comparisons of the spatiotemporal gait and COM variables under with and without obstacle conditions

\begin{tabular}{|c|c|c|c|c|c|c|c|}
\hline \multirow[b]{2}{*}{ Variable } & \multirow{2}{*}{$\begin{array}{l}\text { Obstacle } \\
\text { condition }\end{array}$} & \multicolumn{6}{|c|}{ Pairwise comparison ${ }^{a}$} \\
\hline & & $\begin{array}{l}\text { No task vs. } \\
\text { calling }\end{array}$ & $\begin{array}{c}\text { No task vs. } \\
\text { texting }\end{array}$ & $\begin{array}{c}\text { No task vs. } \\
\text { watching }\end{array}$ & $\begin{array}{c}\text { Calling vs. } \\
\text { texting }\end{array}$ & $\begin{array}{c}\text { Calling vs. } \\
\text { watching }\end{array}$ & $\begin{array}{c}\text { Texting vs. } \\
\text { watching }\end{array}$ \\
\hline \multirow{2}{*}{ Step length } & Without & 0.224 & $0.001^{*}$ & $0.010^{*}$ & 0.094 & 0.410 & 1.000 \\
\hline & With & 1.000 & 0.329 & 0.118 & 0.935 & 0.160 & 1.000 \\
\hline \multirow{2}{*}{ Step time } & Without & 0.141 & $0.001^{*}$ & $0.006^{*}$ & $0.016^{*}$ & 0.602 & 1.000 \\
\hline & With & $0.028^{*}$ & $0.009^{*}$ & $0.023^{*}$ & 0.328 & 0.536 & 1.000 \\
\hline \multirow{2}{*}{ Cadence } & Without & 0.060 & $0.001^{*}$ & $0.003^{*}$ & 0.063 & 1.000 & 1.000 \\
\hline & With & $0.003^{*}$ & $0.004^{*}$ & $0.008^{*}$ & 1.000 & 1.000 & 1.000 \\
\hline \multirow{2}{*}{ Gait speed } & Without & 0.090 & $<0.001^{*}$ & $0.011^{*}$ & $0.006^{*}$ & 0.503 & 0.604 \\
\hline & With & 0.263 & $0.002^{*}$ & $0.030^{*}$ & 0.455 & 0.397 & 1.000 \\
\hline \multirow{2}{*}{$\mathrm{COM}_{\mathrm{ML}}-\mathrm{exc}$} & Without & $0.048^{*}$ & $0.001^{*}$ & 0.064 & 1.000 & 1.000 & 1.000 \\
\hline & With & 0.114 & $0.048^{*}$ & $0.001^{*}$ & 1.000 & 1.000 & 1.000 \\
\hline \multirow{2}{*}{$\mathrm{COM}_{\mathrm{V}}$-exc } & Without & 1.000 & 0.509 & 0.816 & 0.282 & 0.341 & 1.000 \\
\hline & With & $<0.001^{*}$ & $0.037^{*}$ & $0.001^{*}$ & 1.000 & 1.000 & 0.876 \\
\hline \multirow{2}{*}{$\mathrm{COM}_{\mathrm{ML}}$-vel } & Without & $0.008^{*}$ & $0.001 *$ & 0.260 & 1.000 & 1.000 & 0.863 \\
\hline & With & $0.032^{*}$ & 0.124 & $0.024^{*}$ & 1.000 & 1.000 & 1.000 \\
\hline \multirow{2}{*}{$\mathrm{COM}_{\mathrm{V}}$-vel } & Without & 1.000 & 1.000 & 0.840 & 1.000 & 0.107 & 0.572 \\
\hline & With & 1.000 & 1.000 & 1.000 & 1.000 & 1.000 & 1.000 \\
\hline
\end{tabular}

$\mathrm{COM}$ - centre of mass, ML - mediolateral direction, $\mathrm{V}$ - vertical direction, exc - excursion, vel - velocity

a pairwise comparison tested by the post-hoc Bonferroni, * significant difference at $p<0.05$ 

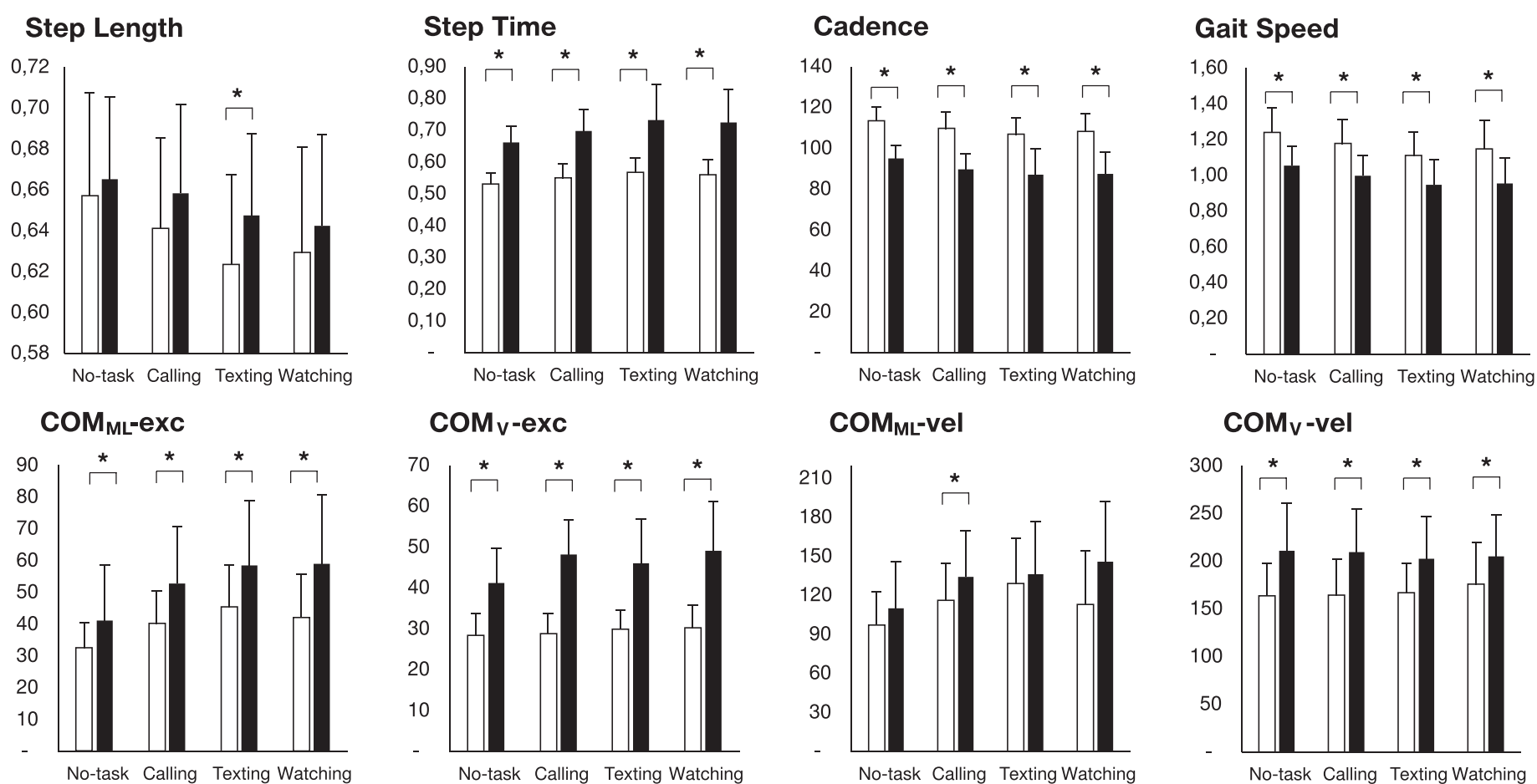

Without obstacle

With obstacle

COM - centre of mass, $\mathrm{ML}$ - mediolateral direction, $\mathrm{V}$ - vertical direction, exc - excursion, vel - velocity Unit of step length is $\mathrm{mm}$, step time is $\mathrm{s}$, cadence is steps/min, gait speed is $\mathrm{m} / \mathrm{s}, \mathrm{COM}_{\mathrm{ML}}$-exc and $\mathrm{COM}_{\mathrm{V}}$-exc is $\mathrm{mm}, \mathrm{COM}_{\mathrm{ML}}-\mathrm{vel}$ and $\mathrm{COM}_{\mathrm{v}}$-vel is $\mathrm{mm} / \mathrm{s}$.

${ }^{*} p<0.05$, significant difference tested by paired $t$-test

Figure 3. Bar charts of data between obstacle conditions

no task and calling $(p<0.001)$, no task and texting $(p$ $=0.037)$, and no task and watching $(p=0.001)$. Regarding $\mathrm{COM}_{\mathrm{ML}}$-vel, significant differences were found between no task and calling $(p=0.032)$ and no task and watching $(p=0.024)$.

Comparison of the spatiotemporal gait and $\mathrm{COM}$ variables between obstacle conditions in each of the smartphone tasks

As shown in Table 3 and Figure 3, significant differences $(p<0.05)$ were observed of all tested variables between obstacle conditions in different smartphone tasks. For no task of smartphone, significant differences in step time $(p<0.001)$, cadence $(p<0.001)$, gait speed $(p<0.001), \mathrm{COM}_{\mathrm{ML}}$-exc $(p=0.024)$, $\mathrm{COM}_{\mathrm{V}}$-exc $(p<0.001)$, and $\mathrm{COM}_{\mathrm{V}}$-vel $(p<0.001)$ were found between with and without obstacle conditions. Concerning calling, significant differences were determined in step time $(p<0.001)$, cadence $(p<0.001)$, gait speed $(p<0.001), \mathrm{COM}_{\mathrm{ML}}$-exc $(p=0.001)$, $\mathrm{COM}_{\mathrm{V}}$-exc $(p<0.001), \mathrm{COM}_{\mathrm{ML}}$-vel $(p=0.032)$, and $\mathrm{COM}_{\mathrm{V}}$-vel, $(p<0.001)$. For texting, significant differences were established in step length $(p=0.002)$, step time $(p<0.001)$, cadence $(p<0.001)$, gait speed $(p<0.001), \mathrm{COM}_{\mathrm{ML}}$-exc $(p=0.009), \mathrm{COM}_{\mathrm{V}}$-exc $(p<$ $0.001)$, and $\mathrm{COM}_{\mathrm{v}}$-vel $(p=0.002)$. Regarding watching, significant differences were noted in step time $(p<$ $0.001)$, cadence $(p<0.001)$, gait speed $(p<0.001)$, $\mathrm{COM}_{\mathrm{ML}}$-vel $(p<0.001), \mathrm{COM}_{\mathrm{ML}}$-exc $(p=0.005)$, and $\mathrm{COM}_{\mathrm{V}}$-vel $(p=0.035)$.

\section{Discussion}

Young adults regularly engage with smartphones in a variety of daily life situations. Recently, a few studies have investigated the impact of smartphone use on confronting obstacle conditions concerning the spatiotemporal gait or COM variables $[9,14,15]$. However, this may have been limited by the smartphone task being tested as considered texting only. The smartphone app has been developed to provide various useful forms in conjunction with changing customer behaviours in their hustle and bustle lifestyles. A smartphone is usually used at all times, whether while in a still position or moving.

As expected, the present findings revealed significant perturbation of smartphone tasks during walk- 
T.E. Susilo, S. Bovonsunthonchai, P. Wattananon, Smartphone use and variables of gait and centre of mass

ing under with or without obstacle conditions in almost all tested variables, except for $\mathrm{COMv}$-vel. The sub-analysis also showed differences in the tested variables between obstacle conditions for each of the smartphone tasks. Furthermore, obstacles significantly influenced each smartphone task, as evidenced by the increased step time, $\mathrm{COM}_{\mathrm{ML}}$-exc, $\mathrm{COM}_{\mathrm{V}}$-exc, and $\mathrm{COM}_{\mathrm{V}}$-vel, as well as decreased cadence and gait speed. However, increased step length was found in texting, and increased $\mathrm{COM}_{\mathrm{ML}}$-vel was observed in calling only. Still, all participants in the study could complete the tasks with sufficient postural stability even when confronted with the challenging distraction by the smartphone and obstacle.

\section{Smartphone distraction}

In different smartphone task comparisons, most alterations could be detected in all spatiotemporal gait and $C O M$ variables. Similar to related studies $[6,11$, 16-19], we found decreased gait speed, step length, and cadence, as well as increased step width when a smartphone was used to perform different tasks. Under the no obstacle condition, significant changes were determined in all spatiotemporal gait variables during texting and watching but without a change in calling. This finding was similar to that in a previous study [19], which revealed a significant reduction of gait speed when a smartphone was used to search for information on the Internet and take a self-portrait, while dialling was not much affected. Reduced cadence was observed while using the smartphone in different tasks for our study. This result is consistent with that in a related study $[6,16]$, while another study showed the opposite outcome, with an increasing value of cadence [19]. The differences in gait variables between studies possibly stem from varied testing and analysis contexts.

For the COM findings, the alteration was more noticeable in both excursion and velocity of the COM in the mediolateral direction in our study. The modification of movement control in terms of walking balance may be related to the changes in spatiotemporal gait variables, especially in reduced gait speed [20]. A greater COM displacement in the mediolateral direction was associated with postural sway stability and falling [14, 20]. The greater degrees of excursion and velocity of COM in the mediolateral direction shown in this study conveyed the disturbance of balance control that occurred while texting and watching. Likewise, a study by Lim et al. [21], investigating the effect of texting over walking on balance pertur- bation revealed a greater margin of stability in walking while texting compared with walking only. With the different methods of examination, another study [22] also determined significant changes in dynamic stability tracked by the trunk kinematics and gait variability in calling and texting. This considerable postural sway was related to gait imbalance, indicating a loss of visuospatial information that proceeded information towards locomotion to plan the route and orientation and maintain dynamic stability [21, 23]. The 'Six Determinants of Gait' theory proposed by Saunders et al. [24] suggested that normal gait control necessitated a particular biomechanical feature of the COM excursion to provide proper metabolic energy consumption while walking. The strategy of increased COM displacement was presented in walking together with smartphone interference to maintain postural stability [22].

Explanations of reduced gait performance while undertaking a dual task as smartphone use were the consequences of visual distraction [15, 21], increased cognitive load [19], loss of attention [8], and reduced capability of information processing [11]. The central pattern generators, with the prominent role of synchronizing limb function during gait, may be loaded by the additional disturbance of smartphone tasks [12]. A review study by Hamacher et al. [25] reported that proper gait function was associated with the complicated cortical and subcortical activation control. When applying more challenging tasks, the neural activations in the cortical and subcortical areas were increased slightly, and integrating these brain areas involved several sensory information inputs from the hippocampus, occipital gyrus, postcentral gyrus, and parietal gyrus [25, 26].

Each smartphone task tested in this study contained a particular channel of distraction following the brain mechanism, as mentioned above. One related study reported that texting interfered with the visual information on the screen [17]. For the protocol of texting investigated in our study, the participants were asked to memorize a brief sentence comprising a combination of words, numbers, and symbols. We assumed that this might require multiple connections and functions in the brain areas to perform the dual-gait task. In calling, possible distraction was attained predominantly from the communication process. Language skill requires the functioning of the frontotemporal cortex, with the most crucial role of speech linked to the Broca area [27, 28]. For watching, this particular task is associated with disturbances of auditory and visual inputs [29,30]. 
Moreover, the restricted arm position at the torso, as shown in calling, watching, and texting, could be another factor affecting the natural gait character $[22,31]$. In this study, one hand held the phone during calling, while 2 hands held the phone during watching, texting, and playing. For texting, this task induced a more coordinated function of thumbs and fingers of both hands, trying to find letters on the screen keys to type the sentence [32]. The coordinated function of multiple body systems requires higher motor brain areas activational control [28]. In brief, all applied smartphone tasks in this study could modify gait performance associated with cognitive distraction [29, 33].

\section{Confronting obstacle challenge}

When engaging with an obstacle, a greater adaptation strategy occurred, as exhibited with a considerable variation of the spatiotemporal gait and COM variables in both with or without using a smartphone. When considering the age group that may be prone to injury from falls or trips, most attention is directed towards the elderly owing to the deteriorating body systems [34, 35]. However, the challenging tasks of smartphone using together with locomotion performing in various environments could usually be observed in young lifestyles. When computing the changes of various gait variables between with and without obstacle conditions among different smartphone tasks, an increase of $2-5 \%$ for step length and an increase of $25-29 \%$ for step time were found. In addition, a decrease of $16-19 \%$ for cadence and a decrease of 12 $15 \%$ for gait speed were determined. For COM variables, an increase of $27-40 \%$ for $\mathrm{COM}_{\mathrm{ML}}$-exc and an increase of $45-68 \%$ for $\mathrm{COM}_{V}$-exc were established. In addition, an increase of $6-29 \%$ for $\mathrm{COM}_{\mathrm{ML}}$-vel and an increase of $17-29 \%$ for $\mathrm{COM}_{\mathrm{V}}$-vel were noted. We compared these percentages of change with a previous study [14], which investigated the effect of texting and crossing obstacles on gait imbalance. Gait speed was decreased by $11 \%$ and COM excursion in mediolateral direction was increased by $17.2 \%$ in the condition of texting with walking and crossing an obstacle compared with walking and crossing an obstacle only. An alteration of gait with a longer walking time was also presented in an earlier study under texting and crossing an obstacle condition [9].

As mentioned above in the context of smartphone distraction, the participants used the working memory load to process the information from the different smartphone tasks. This was additionally challenged by an obstacle that was placed in the middle of the walkway in this study. This obstacle may have distracted visual information because the subjects had to switch to look at the object so that they could cross the obstacle without stumbling or falling. As the categorized dual tasks were challenging, this study set the tasks to provide challenges for both orientation and executive attention. The orientation attentional system was associated with the capability to recognize and prioritize the spatial (location) information, and the executive attentional system involves the ability to decide on the basis of conflicting information [21, 23]. The high executive tasks required adapting a conservative strategy by walking slower to avoid tripping over the obstacle [23]. The last explanation indicates that an initial brain activity occurred before negotiating the obstacle among young adults while operating the smartphone to acquire the environment. In line with a study conducted by Maidan et al. [36], the multi-task walking increased brain activation in the prefrontal area among young adults.

For the results of the balance perturbation in this study, predominantly increased excursions and peak velocities of COM were found in both mediolateral and vertical directions when performing the different smartphone tasks. However, the effect of an obstacle was more noticeable in the vertical than in the mediolateral direction. This observation corroborates related studies [37, 38], reporting that the COM excursion and velocity in the vertical direction were more sensitive because the obstacle directly affected the $d y$ namic stability control while crossing. The COM displacement was elevated in this direction, following the increased foot clearance height to avoid striking the obstacle.

When implementing obstacle crossing together with different smartphone tasks, modification of gait showed alteration of the COM excursion rather than velocity in the mediolateral direction. This may be related to the disruption of cognitive performance when performing the dual task. Similarly, a study by Chen et al. [14] revealed postural sway in the mediolateral direction when texting and crossing an obstacle. In addition, the limited visual feedback triggered greater facilitation of cutaneous reflex activation of the biceps femoris, which is responsible for greater knee flexion to avoid the obstacle [39]. This showed evidence of movement adaptation to perform the tasks successfully.

In brief, among the healthy young adults who participated in this study, we found movement changes that involved the perspective of the spatiotemporal gait and COM variables. It should be noted that the participants were the most familiar with a smartphone 
T.E. Susilo, S. Bovonsunthonchai, P. Wattananon, Smartphone use and variables of gait and centre of mass

and able to adapt themselves to perform several tasks concurrently.

\section{Limitation of the study}

This study may be limited by the tested population, comprising healthy young adults only. All individuals were experienced in using a smartphone for many years and used it frequently. Other groups of less experienced subjects or other age groups may be of interest for further research. In addition, evaluation in real-world situations and with other types of gait and balance variables should be applied to provide more information.

\section{Conclusions}

This study indicated that the spatiotemporal gait and $\mathrm{COM}$ variables among young adults were predominantly altered by concurrent smartphone tasks confronted by obstacle crossing. Cognitive distraction played the prominent role in the modification of walking while performing different smartphone tasks, and the obstacle condition affected the biomechanical and motor control strategy alteration, as implied by the observed changes in COM excursion and velocity. These results suggest that young adults should be aware of gait and balance disturbances resulting from smartphone use while performing daily mobility activities.

\section{Acknowledgements}

This study was partially granted by a Mahidol University Graduate Alumni Association scholarship. The authors would like to thank the Faculty of Physical Therapy, Mahidol University for providing data collection space, equipment, and support. The authors also would like to thank the Gait and Posture Laboratory members who helped in the data collection process. The most important persons were all participants who volunteered to participate in the study.

\section{Disclosure statement}

No author has any financial interest or received any financial benefit from this research.

\section{Conflict of interest}

The authors state no conflict of interest.

\section{References}

1. Csibi S, Griffiths MD, Demetrovics Z, Szabo A. Analysis of problematic smartphone use across different age groups within the 'components model of addiction'.
Int J Ment Health Addiction. 2021;19:616-631; doi: 10.1007/s11469-019-00095-0.

2. Kim H-J, Min J-Y, Kim H-J, Min K-B. Accident risk associated with smartphone addiction: a study on university students in Korea. J Behav Addict. 2017;6(4): 699-707; doi: 10.1556/2006.6.2017.070.

3. Kim Y, Briley DA, Ocepek MG. Differential innovation of smartphone and application use by sociodemographics and personality. Comput Hum Behav. 2015;44:141147; doi: 10.1016/j.chb.2014.11.059.

4. Schade SA, Mahoney JM, Spotts AV, Greenauer N, Veerabhadrappa P. Pokémon Go did not increase step count or distance travelled among college students. Hum Mov. 2020;21(2):64-70; doi: 10.5114/hm.2020.89916.

5. Derhon V, Santos RA, Brandalize M, Brandalize D, Rossi LP. Intra- and inter-examiner reliability in angular measurements of the knee with a smartphone application. Hum Mov. 2017;18(2):38-43; doi: 10.1515/ humo-2017-0011.

6. Crowley P, Madeleine P, Vuillerme N. The effects of mobile phone use on walking: a dual task study. BMC Res Notes. 2019;12(1):352; doi: 10.1186/s13104-0194391-0.

7. Zhang L, Cui B, Yang M, Guo F, Wang J. Effect of using mobile phones on driver's control behavior based on naturalistic driving data. Int J Environ Res Public Health. 2019;16(8):1464; doi: 10.3390/ijerph16081464.

8. Lin M-IB, Huang Y-P. The impact of walking while using a smartphone on pedestrians' awareness of roadside events. Accid Anal Prev. 2017;101:87-96; doi: 10.1016/j.aap.2017.02.005.

9. Licence S, Smith R, McGuigan MP, Earnest CP. Gait pattern alterations during walking, texting and walking and texting during cognitively distractive tasks while negotiating common pedestrian obstacles. PLoS One. 2015;10(7):e0133281; doi: 10.1371/journal.pone.013 3281.

10. Hyman IE Jr, Boss SM, Wise BM, McKenzie KE, Caggiano JM. Did you see the unicycling clown? Inattentional blindness while walking and talking on a cell phone. Appl Cogn Psychol. 2010;24(5):597-607; doi: 10.1002/acp.1638.

11. Stöckel T, Mau-Moeller A. Cognitive control processes associated with successful gait performance in dual-task walking in healthy young adults. Psychol Res. 2020; 84(6):1766-1776; doi: 10.1007/s00426-019-01184-4.

12. Shumway-Cook A, Woollacott MH. Motor control: translating research into clinical practice, $5^{\text {th }} \mathrm{ed}$. Philadelphia: Lippincott Williams \& Wilkins; 2017.

13. Tesio L, Rota V. The motion of body center of mass during walking: a review oriented to clinical applications. Front Neurol. 2019;10:999; doi: 10.3389/fneur.2019. 00999.

14. Chen S-H, Lo O-Y, Kay T, Chou L-S. Concurrent phone texting alters crossing behavior and induces gait imbalance during obstacle crossing. Gait Posture. 2018; 62:422-425; doi: 10.1016/j.gaitpost.2018.04.004. 
15. Chopra P, Castelli DM, Dingwell JB. Cognitively demanding object negotiation while walking and texting. Sci Rep. 2018;8(1):17880; doi: 10.1038/s41598-01836230-5.

16. Bovonsunthonchai S, Ariyaudomkit R, Susilo TE, Sangiamwong $\mathrm{P}$, Puchaphan $\mathrm{P}$, Chandee S, et al. The impact of different mobile phone tasks on gait behaviour in healthyyoung adults. J Transp Health. 2020;19:100920; doi: 10.1016/j.jth.2020.100920.

17. Plummer P, Apple S, Dowd C, Keith E. Texting and walking: effect of environmental setting and task prioritization on dual-task interference in healthy young adults. Gait Posture. 2015;41(1):46-51; doi: 10.1016/j. gaitpost.2014.08.007.

18. Agostini V, Lo Fermo F, Massazza G, Knaflitz M. Does texting while walking really affect gait in young adults? J Neuroeng Rehabil. 2015;12:86; doi: 10.1186/s129 84-015-0079-4.

19. Niederer D, Bumann A, Mühlhauser Y, Schmitt M, Wess K, Engeroff T, et al. Specific smartphone usage and cognitive performance affect gait characteristics during free-living and treadmill walking. Gait Posture. 2018;62:415-421; doi: 10.1016/j.gaitpost.2018.04.007.

20. Orendurff MS, Segal AD, Klute GK, Berge JS, Rohr ES, Kadel NJ. The effect of walking speed on center of mass displacement. J Rehabil Res Dev. 2004;41(6A):829_ 834; doi: 10.1682/jrrd.2003.10.0150.

21. Lim J, Chang SH, Lee J, Kim K. Effects of smartphone texting on the visual perception and dynamic walking stability. J Exerc Rehabil. 2017;13(1):48-54; doi: 10.12965/jer.1732920.460.

22. Magnani RM, Lehnen GC, Rodrigues FB, de Sá E Souza GS, de Oliveira Andrade A, Vieira MF. Local dynamic stability and gait variability during attentional tasks in young adults. Gait Posture. 2017;55:105-108; doi: 10.1016/j.gaitpost.2017.04.019.

23. Lo O-Y, Chou L-S. Effects of different visual attention tasks on obstacle crossing in healthy young adults. BiomedEng ApplBasis Commun.2015;27(6):1550059; doi: 10.4015/s1016237215500593.

24. Saunders JB, Inman VT, Eberhart HD. The major determinants in normal and pathological gait. J Bone Joint Surg Am. 1953;35-A(3):543-558.

25. Hamacher D, Herold F, Wiegel P, Hamacher D, Schega L. Brain activity during walking: a systematic review. Neurosci Biobehav Rev. 2015;57:310-327; doi: 10.1016/ j.neubiorev.2015.08.002.

26. Fuster JM. Prefrontal cortex. In: Squire LR (ed.), Encyclopedia of neuroscience. Oxford: Academic Press; 2009; 905-908.

27. Jeon S, Kim C, Song S, Lee G. Changes in gait pattern during multitask using smartphones. Work. 2015;53(2): 241-247; doi: 10.3233/WOR-152115.

28. Pizzamiglio S, Naeem U, Abdalla H, Turner DL. Neural correlates of single- and dual-task walking in the real world. Front Hum Neurosci. 2017;11:460; doi: 10.3389/ fnhum.2017.00460.
29. Sejdić E, Findlay B, Merey C, Chau T. The effects of listening to music or viewing television on human gait. Comput Biol Med. 2013;43(10):1497-1501; doi: 10.1016/ j.compbiomed.2013.07.019.

30. Stoffregen TA, Pagulayan RJ, Bardy BG, Hettinger LJ. Modulating postural control to facilitate visual performance. Hum Mov Sci. 2000;19(2):203-220; doi: 10.1016/S0167-9457(00)00009-9.

31. Yang HS, Atkins LT, Jensen DB, James CR. Effects of constrained arm swing on vertical center of mass displacement during walking. Gait Posture. 2015;42(4): 430-434; doi: 10.1016/j.gaitpost.2015.07.010.

32. Xiong J, Muraki S. An ergonomics study of thumb movements on smartphone touch screen. Ergonomics. 2014;57(6):943-955; doi: 10.1080/00140139.2014.90 4007.

33. Timmis MA, Bijl H, Turner K, Basevitch I, Taylor MJD, van Paridon KN. The impact of mobile phone use on where we look and how we walk when negotiating floor based obstacles. PLoS One. 2017;12(6):e0179802; doi: 10.1371/journal.pone.0179802.

34. Da Rocha ES, Machado ÁS, Franco PS, Guadagnin EC, Carpes FP. Gait asymmetry during dual-task obstacle crossing in the young and elderly. Hum Mov. 2013; 14(2):138-143; doi: 10.2478/humo-2013-0016.

35. Uchiyama M, Demura S, Sugiura H. The mobility performance of the elderly before, during and after crossing over an obstacle. Hum Mov. 2012;13(4):297-302; doi: 10.2478/v10038-012-0034-1.

36. Maidan I, Shustak S, Sharon T, Bernad-Elazari H, Geffen N, Giladi N, et al. Prefrontal cortex activation during obstacle negotiation: what's the effect size and timing? Brain Cogn. 2018;122:45-51; doi: 10.1016/j. bandc.2018.02.006.

37. Chou LS, Kaufman KR, Brey RH, Draganich LF. Motion of the whole body's center of mass when stepping over obstacles of different heights. Gait Posture. 2001; 13(1):17-26; doi: 10.1016/s0966-6362(00)00087-4.

38. Wang T-M, Chen H-L, Lu T-W. Effects of obstacle height on the control of the body center of mass motion during obstructed gait. J Chin Inst Eng. 2007;30(3):471479; doi: 10.1080/02533839.2007.9671275.

39. Marigold DS, Chang AJ, Lajoie K. Cutaneous reflex modulation during obstacle avoidance under conditions of normal and degraded visual input. Exp Brain Res. 2017;235(8):2483-2493; doi: 10.1007/s00221017-4976-6. 